\title{
Vignettes
}

\section{Comparing Outcomes for Undergraduate Psychology Research Assistants and Nonresearchers}

\author{
Laura A. Pawlow and Elizabeth J. Meinz \\ Southern Illinois University Edwardsville, Ipawlow@siue.edu
}

\section{doi: $10.18833 /$ spur/1/3/1}

Undergraduate research assistants (RAs) in psychology engage in literature review, experimental design, IRB submission, data collection, entry, and analysis, as well as craft summaries of results. Faculty mentors also typically serve as informal advisers on such issues as career paths, graduate school, and professionalism. This study investigated whether psychology RAs were distinguishable from undergraduates who did not serve as research assistants (non-RAs) and utilized a pretest-posttest design and a control group, which is relatively rare in the assessment of undergraduate research outcomes.

A total of 178 psychology students completed the department's Curriculum Assessment Battery (CAB) both as a pretest (within one semester of declaring as a major) and a posttest (during senior year). The multiple-choice questions measured Career Knowledge (CK), Statistics/Methods Knowledge (SM), Core Knowledge from required courses (Core), Critical Thinking in Psychology (CT; modified from Lawson 1999), and Writing for Psychology (WR), as well as two self-report Likert questions that measured knowledge of PowerPoint (PP) and SPSS.

Forty-two percent of the sample had participated as RAs. RAs did not significantly differ from non-RAs in gender, age, or race but had significantly higher ACT scores and cumulative GPAs. Mixed-model ANOVAs revealed that both groups performed significantly better at posttest than pretest. In addition, RAs significantly outperformed non-RAs on the variables of SM, WR, CK, CT, and Core ( $p<.01$ for all significant effects). However, no significant interactions were found; that is, the gain from pretestposttest was similar for RAs and non-RAs. Additionally, follow-up analyses to determine whether the benefits of research participation across time were greater for lowthan for high-ability students (dichotomized ACT scores and GPAs) again revealed no significant interactions.

These data suggest that undergraduate researchers have overall higher academic abilities and knowledge than nonresearchers, suggesting that either more academically able students self-select into RA experiences or that faculty choose such students based on their academic records.
This may suggest that faculty should open their labs to students with a broader ability profile if the benefits of participation are to be spread equitably among all students. However, these data also suggested no direct benefit of serving as an RA on psychology skills/knowledge, thus challenging previous research that demonstrated academic gains directly related to research participation (e.g., Lopatto 2009). It is important to note, however, that these findings may be unique to this particular, psychology-based assessment tool, which may not capture more generalized research-based skills and knowledge as more commonly reported in the literature.

\section{References}

Lawson, Timothy. 1999. "Assessing Psychological Critical Thinking as a Learning Outcome for Psychology Majors." Teaching of Psychology 26: 207-209.

Lopatto, David. 2010. "Undergraduate Research as a High-Impact Student Experience.” Peer Review 12(2): 27-30.

\section{Undergraduate Research and Student Success: A Model for Cultural Competency as a Mediator}

\author{
Jacob Alan English and Christina Wan \\ Georgia State University, jacobenglish@gsu.edu
}

\section{doi: 10.18833/spur/1/3/2}

Undergraduate research (UR) has proven to be a high-impact educational practice that contributes to student success. Additionally, teaching cultural competence is an important factor in enhancing students' academic and behavioral outcomes. Although there is a wealth of knowledge on the benefits of UR participation, there is a lack of information about incorporating cultural competencies in UR experiences to further enhance student success. The University Assistantship Program (UAP) at Georgia State University wanted to explore the effects of an undergraduate research experience focused on developing cultural competencies on student success. The UAP matches students with faculty and staff to work on research and career-related projects in alignment with students' professional goals. In 2016, the program partnered with the Multicultural Center, a unit of Student Affairs, to establish University Assistant (UA) positions within its office. The partnership set out to (1) explore the relationship among undergraduate research, cultural competence, and student success; (2) intentionally develop cultural competence among students through programming and mentoring; and (3) develop a model for other UAP partners to follow. The Council on Undergraduate Research's 
strategic pillar on diversity and inclusion in undergraduate research was the basis for the model. The hope in presenting this pilot is to provide the undergraduate research community with an innovative model to increase participation in undergraduate research by offering diverse and inclusive research experiences.

The Multicultural Center UAs engage in hands-on diversity and inclusion work, which provides them with a platform for later development of their own research projects. A central understanding of these positions is that the students' research projects may represent diversity in both topic and format, with students producing research projects in both traditional and nontraditional forms. The UAs serve as research assistants for a study on cultural competence on campus; work on projects such as the Community Conversations workshops where they assist in facilitating conversations with students about diversity, inclusion, and social justice; assist with developing lesson plans for a cultural competence course taught for credit on campus; and coordinate programs in faculty and staff mentorship as well as peer mentorship. Through the UAP, they can collaboratively develop programming and outreach to serve diverse students, through which they increase their own cultural competence. The program also assesses students' attainment of cultural competency through a performance evaluation process that examines the effects of the research experience and engagement in content concerning differentness, analyzing their role in student success.

\section{Engaging Academically At-Risk Student Populations in Research: Initial Assessment of an Undergraduate Research Certificate Program}

Frances Shen, Keenan Dungey, Brian Kahn, John Laubersheimer, Tarah Sweeting-Trotter, Alexander Skarr, Sudeep

Sharma, Ben Walsh, and Holly Kent

University of Illinois Springfield, fshen3@uis.edu

\section{doi: $10.18833 /$ spur/1/3/3}

Since the University of Illinois Springfield (UIS) has been successful at recruiting a larger and more diverse undergraduate population, including academically at-risk students, more effort is needed to ensure their academic success. Thus, in collaboration with the Center for Academic Success in the Division of Academic Affairs, the Undergraduate Research Support program developed an Undergraduate Research Certificate program, seeking to encourage academically underprepared students to engage in undergraduate research by providing training and support services. The program is in the pilot stage with first-year students in the Students Transitioning for Academic Retention and Success (STARS) living-learning community. STARS serves an academically underprepared student population that typically consists of minorities and first-generation college students.

In the freshmen seminar course, STARS students participate in a number of workshops that address topics such as the definition of research, benefits of engaging in undergraduate research, undergraduate research resources and opportunities at UIS, presentation skills, information literacy skills, writing and citation skills, and so forth. Students also complete additional workshops outside of the course to receive the undergraduate research certificate. This requirement helps ensure that only students who are intrinsically motivated to engage in undergraduate research receive the certificate and matriculate into research opportunities that involve direct work with faculty members.

The program expressly involves undergraduate students who have actively engaged in research across various disciplines, as they participate as workshop presenters. This particular approach serves to (1) heighten students' interest in and motivation for undergraduate research through peer explanations of benefits and enthusiasm about research, (2) increase students' self-efficacy by witnessing peer success in undergraduate research, (3) enhance the visibility of student researchers on campus, and (4) involve students in a culture of undergraduate research engagement.

Pretest and posttests were administered to examine student perceptions of research self-efficacy, perceived barriers, and interest in undergraduate research. Results from the pretest confirm that undergraduate students often lack selfconfidence, awareness of opportunities, and the necessary skills to foster involvement in undergraduate research. Limited posttest data suggests the certificate program had a minor, positive effect on student perceptions. However, more data are needed to assess the effectiveness of the program. In the future, tracking student participation in undergraduate research after completion of the certificate is planned, as well as comparing outcomes for these students with those of similar academic backgrounds who do not participate in the program.

\section{Increasing Potential and Widening Horizons: Promoting Undergraduate Research at a Two-Year Regional Campus}

\author{
Elizabeth Easley and Sarah Hunt Sellhorst \\ University of South Carolina Lancaster, Sehunt@mailbox.sc.edu
}

\section{doi: $10.18833 /$ spur/1/3/4}

The University of South Carolina Lancaster (USCL)'s Research Club is a student organization established in 2015 as part of efforts to nurture a culture of scholarly 